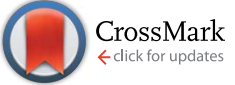

Cite this: Chem. Sci., 2016, 7, 7013

\section{Emissive nanoparticles from pyridinium- substituted tetraphenylethylene salts: imaging and selective cytotoxicity towards cancer cells in vitro and in vivo by varying counter anions $\uparrow$}

\author{
Yanyan Huang, ${ }^{\text {ab }}$ Guanxin Zhang, ${ }^{\text {ab }}$ Fang Hu, ${ }^{\text {ab }}$ Yulong Jin, ${ }^{\text {ab }}$ Rui Zhao *ab \\ and Deqing Zhang*ab
}

\begin{abstract}
Chemotherapeutics specifically targeting cancer cells without damaging healthy cells is the long-awaited goal of cancer treatment. In this paper, a series of nanoparticles (NanoTPES 1-4) assembled from pyridinium-substituted tetraphenylethylene salts were synthesized and investigated both in vitro and in vivo for this purpose. By changing the counter anions, NanoTPES 1-4 exhibit tunable emission colors, sizes and surface charges. NanoTPES 2 and 3 with tetraphenyl borate and tetra(4-chlorophenyl) borate as the respective anions selectively imaged and targeted mitochondria in cancer cells. Accordingly, these two nanoparticles specifically kill cancer cells with minimal effect on normal cells. Such selective cytotoxicity was attributed to the change of membrane potential and inhibition of ATP synthesis in the mitochondria of cancer cells. Furthermore, both NanoTPES 2 and 3 exhibited efficient tumor accumulation and tumor growth inhibition in vivo, with negligible systemic toxicity.
\end{abstract}

range of surface chemistries, and thus tuning the surface properties, become ideal candidates to realize targeting, imaging and therapeutic functions simultaneously. ${ }^{7}$

Tetraphenylethylene (TPE) is known as one of the aggregation-induced emission (AIE) fluorogens. ${ }^{8}$ By manipulating the aggregation and deaggregation of TPE molecules, chemo-/ biosensors have been successfully constructed. ${ }^{9}$ TPE molecules with targeting groups have also been utilized for cell and organelle bioimaging and phototherapy. ${ }^{\mathbf{1 0}}$ Moreover, TPE molecules in poor solvents can assemble into nanoparticles, and the emissive dots formed in the presence of amphiphiles have been employed for bioimaging. ${ }^{\mathbf{1 0 a 1 1}}$ However, selective cytotoxicity of TPE molecules towards cancer cells has never been reported.

In this work, we report nanoparticles self-assembled from pyridinium-substituted tetraphenylethylene with different counter anions (NanoTPES 1-4, Scheme 1). The sizes and surface zeta-potentials of NanoTPES 1-4 can be tuned by varying the counter ions. Interestingly, it was discovered that these nanoparticles interact with normal and cancer cells differently. The cytotoxicity of the nanoparticles can be tuned by the respective counter anions. The in vivo anti-cancer activity of NanoTPES was also examined with tumor-bearing mice. The targeting of NanoTPES 2 and $\mathbf{3}$ (with tetraphenyl borate and tetra(4-chlorophenyl) borate as the respective anions) to the tumor site facilitated the selective accumulation, efficacious tumor inhibition and minimal systemic cytotoxicity.
${ }^{a}$ Beijing National Laboratory for Molecular Sciences, CAS Key Laboratories of Organic Solids and Analytical Chemistry for Living Biosystems, Institute of Chemistry, Chinese Academy of Sciences, Beijing, 100190,China.E-mail:dqzhang@iccas.ac.cn; zhaorui@ iccas.ac.cn; gxzhang@iccas.ac.cn

${ }^{b}$ University of Chinese Academy of Sciences, Beijing 100049, China

$\dagger$ Electronic supplementary information (ESI) available. See DOI: 10.1039/c6sc02395a 


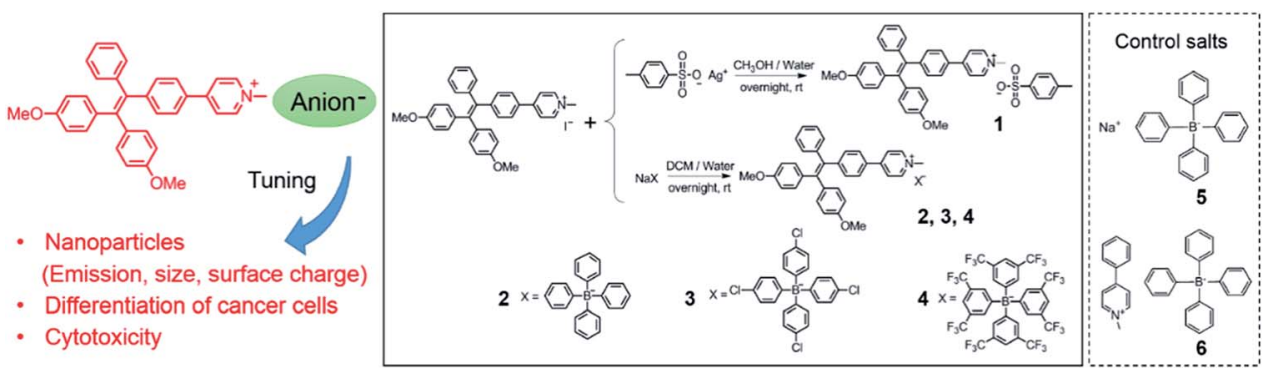

Scheme 1 The chemical structures of compounds 1-6 and the synthetic approach.

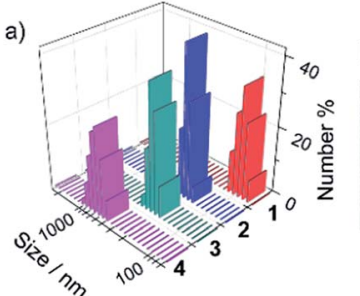

b)
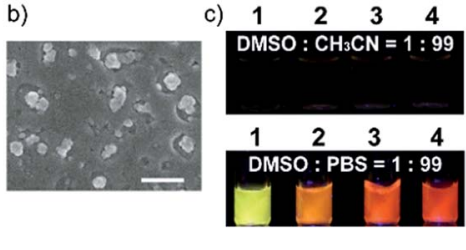

Fig. 1 Fluorescence and aggregation behavior. (a) Size and distribution of NanoTPES derived from DLS data; (b) SEM image of NanoTPES 2, the scale bar was $500 \mathrm{~nm}$; (c) photographs of four compounds in different solvents under UV light $(365 \mathrm{~nm})$ irradiation.

Table 1 Sizes and zeta potentials of NanoTPES

\begin{tabular}{llc}
\hline NanoTPES & $\begin{array}{l}\text { Average size } \\
(\mathrm{nm})\end{array}$ & $\begin{array}{l}\text { Zeta potential } \\
(\mathrm{mV})\end{array}$ \\
\hline $\mathbf{1}$ & 108 & 0.7 \\
2 & 219 & 30.4 \\
$\mathbf{4}$ & 255 & 36.2 \\
& 609 & -9.9
\end{tabular}

\section{Results and discussion}

\section{Molecular design, preparation and characterization of the nanoparticles}

The unusually hyperpolarized mitochondrial membrane potential is emerging as a bioelectric sign of cancer cells. ${ }^{12}$ Owing to the vital roles of mitochondria in programmed cell death and power supply of cells, ${ }^{13}$ targeting agents capable of destroying mitochondria are effective in killing cancer cells. ${ }^{14}$ Herein, based on the fact that cationic compounds have the tendency to accumulate in mitochondria, ${ }^{15}$ pyridinium was linked to TPE to introduce delocalized positive charge. The derivatization of the pyridinium group is also intended to shift the blue emission of TPE to the long wavelength region, which is appealing for bioimaging. Counter anions with different hydrophobicities were chosen, including toluenesulfonate, tetraphenyl borate, tetra(4-chlorophenyl) borate and tetra(3,5bis(trifluoromethyl)phenyl) borate (Scheme 1). Compounds 1-4 were prepared using ion-exchange procedures starting from the corresponding salt with iodide. Their chemical structures were characterized with NMR and FT-IR. The purities were confirmed by elemental analysis (Fig. S1-S4, ESI†).
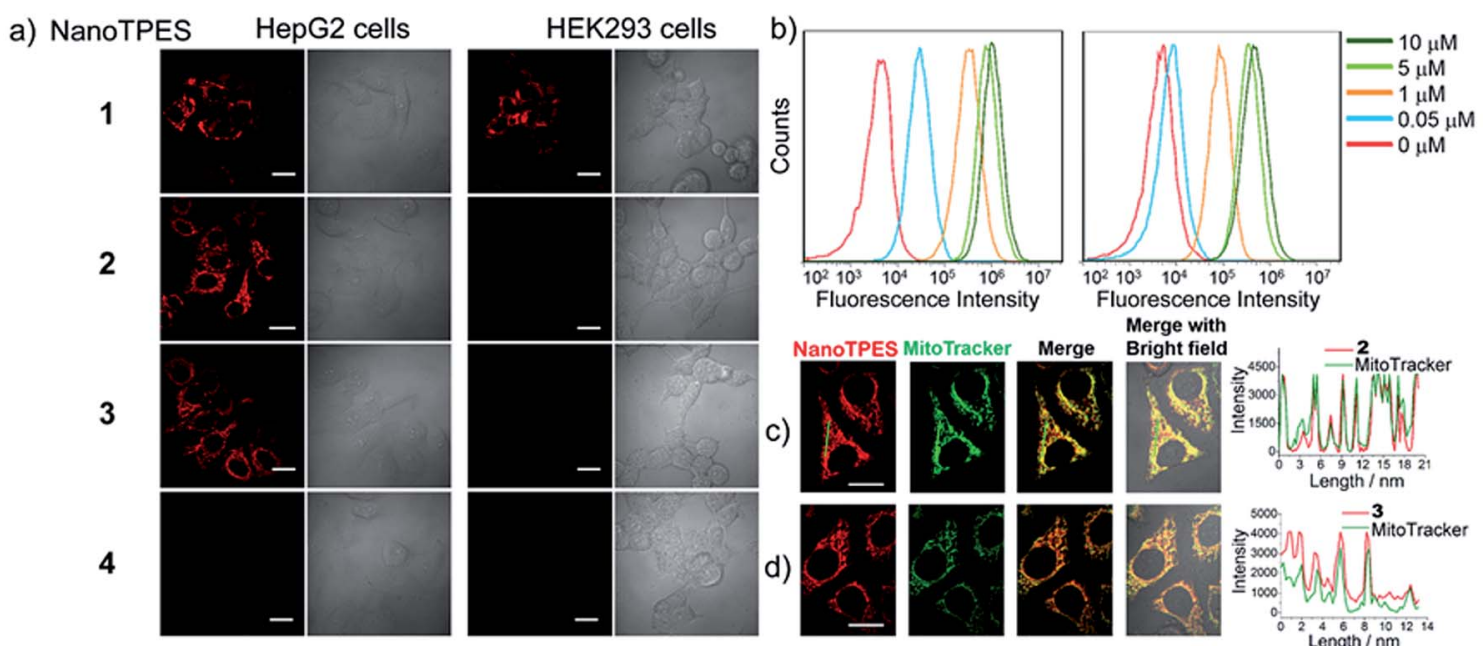

Fluorescence Intensity
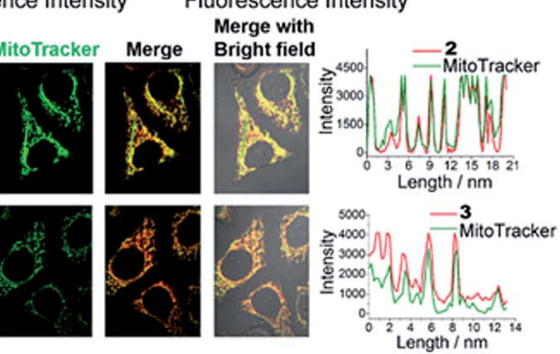

Fig. 2 (a) CLSM images of HepG2 cells and HEK293 cells after treatment with NanoTPES 1-4; scale bar: 20 um; (b) flow cytometric analysis of HepG2 cells after stained with NanoTPES 2 (left) and NanoTPES 3 (right) of different concentrations; (c and d) co-staining of NanoTPES 2 (c) and NanoTPES 3 (d) with mitochondrial tracker Green in HepG2 cells; scale bar: $20 \mu \mathrm{m}$. NanoTPES concentration: $20 \mu \mathrm{M}$. 


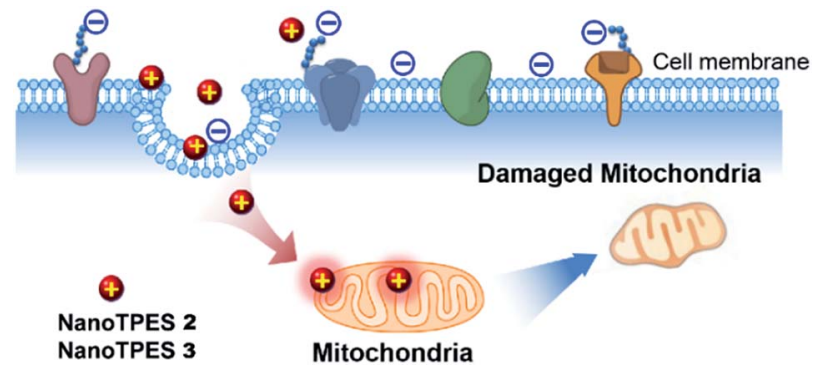

Scheme 2 Illustration of possible pathway of NanoTPES 2 and 3 for targeting and damaging mitochondria in cancer cells.

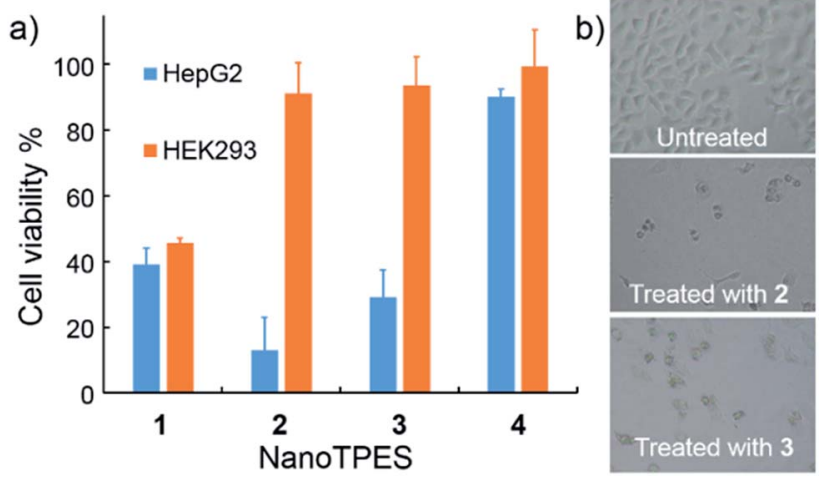

Fig. 3 (a) Cytotoxicity of NanoTPES 1-4 (10 $\mu \mathrm{M}$ each) towards HepG2 cells and HEK293 cells after $48 \mathrm{~h}$ treatment; (b) microscopic observation of HepG2 cells without and with the treatment of NanoTPES 2 and 3 .

The nanoparticles were prepared by adding $10 \mu \mathrm{L}$ of DMSO solutions of compounds 1-4 separately to $990 \mu \mathrm{L}$ of phosphate buffer saline (PBS), and the resulting nanoparticles were referred to as NanoTPES 1, NanoTPES 2, NanoTPES 3 and NanoTPES 4, respectively. On the basis of DLS (dynamic light scattering) data (Fig. 1a), the average sizes of NanoTPES 1-4 were estimated to be $108 \mathrm{~nm}, 219 \mathrm{~nm}, 255 \mathrm{~nm}$ and 609 $\mathrm{nm}$, respectively (Table 1). The resulting nanoparticles were also characterized with scanning electron microscopy (SEM) (Fig. $1 \mathrm{~b}$ and $\mathrm{S} 6 \dagger$ ). The size difference of these nanoparticles is expected to arise from the different hydrophobic properties of the counter anions, which lead to different self-assembled structures. Apart from sizes, the surface charges reflected by zeta-potentials of these nanoparticles can also be tuned by varying the counter anions. As listed in Table 1, the surfaces of NanoTPES 2 and 3 are significantly positively charged, whereas the surface of NanoTPES 4 becomes negatively charged. The zeta-potential of NanoTPES $\mathbf{1}$ is just slightly positive.

Compounds 1-4 were weakly emissive in good solvents such as DMSO and acetonitrile, but became strongly fluorescent in phosphate buffer (Fig. 1c). The fluorescence quantum yields of NanoTPES 1-4 $(20 \mu \mathrm{M})$ in PBS containing $1 \%$ DMSO were measured to be $24.1 \%, 16.5 \%, 26 \%$ and $25.1 \%$, respectively. Interestingly, the emission colors were found to be dependent on the counter anions as shown in Fig. 1c; the emission maximum was shifted from $560 \mathrm{~nm}$ for NanoTPES 1, to $605 \mathrm{~nm}, 620 \mathrm{~nm}$ and $640 \mathrm{~nm}$ for NanoTPES 2, 3 and 4, respectively (Fig. S7 and S8, ESI $\dagger$ ). Thus, NanoTPES 2, 3 and 4 became orange-red or red-emissive when the counter anions were replaced by tetraphenyl borate, tetra(4-chlorophenyl) borate and tetra(3,5-bis(trifluoromethyl)phenyl) borate. The different emission colors of these nanoparticles may be attributed to their different self-assembled structures, within which the cation-anion interactions are different owing to the different electronic structures and sizes of the counter anions. In addition, it is expected that the bulky anions in NanoTPES 2, 3 and 4 may allow the tetraphenylethylene frameworks to possess more planar conformations, which can lead to the red-shifts of the emission according to our previous report. ${ }^{16}$

\section{Cell imaging}

The bright fluorescence of NanoTPES 1-4 enables the monitoring of their interactions with different cells. These nanoparticles were allowed to interact with cancer cells including HepG2 (hepatoblastoma cell line), HeLa (cervical cancer cell line) and A549 (human lung adenocarcinoma epithelial cells), and normal cells including HEK293 (from normal human kidney), Chang liver and L02 (human hepatic cell lines). From CLSM (confocal laser scanning microscopy) images, both cancer (HepG2, HeLa and A549) and normal (HEK293, Chang liver and L02) cells were fluorescent after incubation with NanoTPES 1 (Fig. 2a and S9†), indicating the non-selective uptake of NanoTPES 1 by cancer and normal cells. In comparison, bright red fluorescence was only detected for HepG2, HeLa and A549 cells after incubation with NanOTPES 2 and 3 (Fig. 2a and S9†), whereas no fluorescence was observed for HEK293, Chang liver and L02 cells with the same treatment. The selective imaging suggests the good permeability of NanoTPES 2 and 3 into cancer cells, and their disability to penetrate normal cells. Interestingly, no fluorescence was detected for both cancer and normal cells after incubation with NanoTPES 4 (Fig. 2a and S9†), revealing that the cellular uptake of NanoTPES 4 had not taken place for either cancer or normal cells.

The intracellular delivery of nanoparticles is usually via an endocytosis/phagocytosis pathway in which interactions with the cell membrane may be involved. ${ }^{17}$ Compared with those of normal cells, membranes of cancer cells possess significantly negative charges due to the presence of more anionic biomolecules. ${ }^{18}$ Thus, the selectivity of NanoTPES 2 and 3 may be related to the sizes and surface charges of these nanoparticles. As listed in Table 1, the surfaces of NanoTPES 2 and 3 are more positively charged; accordingly, they can interact with cancer cells more strongly. In addition, the hydrophobic TPE fragment in NanoTPES 2 and 3 can facilitate the uptake and interaction with lipid bilayers of cancer cells, which resulted in their efficient internalization into cancer cells. With regards to normal cells, the absence of the essential electrostatic interaction dramatically weakens the interaction and uptake of NanoTPES 2 and 3 into cells. The significant 

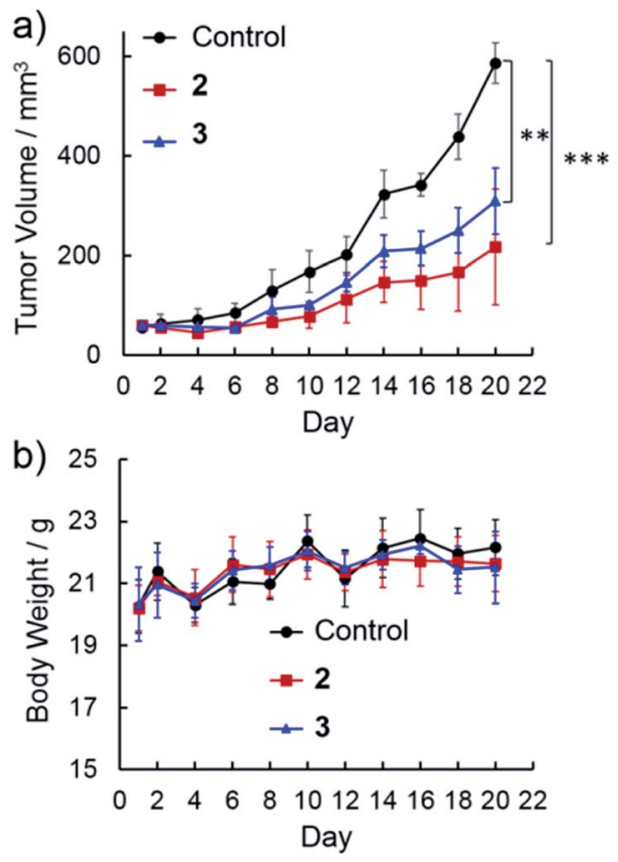

Fig. 4 Potent antitumor activity of NanoTPES via intratumor injection in a tumor xenograft mouse model: (a) variation of the tumor volumes up to day 20 after treatment with NanoTPES 2 and 3 (mean \pm s.d., $n=$ 5) $(* * * P<0.001 ; * * P<0.01$; Student's $t$-test); (b) variation of the body weight of mice up to day 20 after treatment with NanoTPES 2 and 3.

differences in size and surface charge of NanoTPES 1 and 4, in comparison with NanoTPES 2 and 3, can explain the observation that they cannot specifically target cancer cells (see page s18 in $\mathrm{ESI} \dagger)$.

On the basis that NanoTPES 2 and 3 can selectively target cancer cells, flow cytometry, a powerful tool for quantitation, was also used to analyze stained cells. As depicted in Fig. 2b, gradual fluorescence enhancement was observed from HepG2 cells as the concentrations of NanoTPES 2 and $\mathbf{3}$ increased, indicating the continuous uptake of these two nanoparticles. The saturation uptake behaviors of NanoTPES 2 and 3 by HepG2 cells confirm their high affinity and specificity toward cancer cells (Fig. S10, ESI $\dagger$ ). In addition, a time-course assay clearly demonstrated the intracellular trafficking of the nanoparticles from cell membrane into cytoplasm (see page s19 in ESI $\dagger$ ). After incubation for $60 \mathrm{~min}$, a very clear target-to-background image was acquired (Fig. S11 $\dagger$ ), indicating the completion of subcellular localization. As examined by fluorescence colocalization assay, NanoTPES 2 and 3 gave high coefficient values of 0.94 and 0.91 with a commercial mitochondrial tracker (MitoTracker Green) (Fig. 2c and d), demonstrating their good localization to mitochondria. No overlapping was detected with fluorescent trackers for other organelles (such as lysosomes and endosomes) and the cell nucleus (Fig. S12 and S13, ESI $\dagger$ ). Based on the fact that cancer cells usually contain more mitochondria and their mitochondrial membrane is unusually negatively charged, ${ }^{\mathbf{1 2}, 15 b}$ the possible pathway for NanoTPES 2 and 3 to selectively target mitochondria in cancer cells is illustrated in Scheme 2 .

\section{Cytotoxicity tuned by counter anions}

The cytotoxicity of NanoTPES 1-4 towards both cancer cells and normal cells was assayed with the standard MTT (3-(4,5-dimethylthiazol-2-yl)-2,5-diphenyltetrazolium bromide) method. As shown in Fig. 3a, the viability of HepG2 cells was measured to be only $13 \%$ and $29 \%$ respectively, after treatment with NanoTPES 2 and 3 for 48 h. Accordingly, HepG2 cells also showed typical morphology of dead cells, being shrunken, rounded up and detached after incubation with either

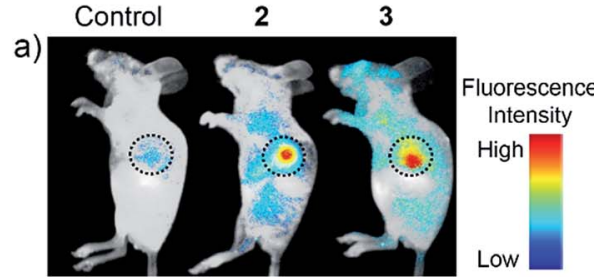

C)

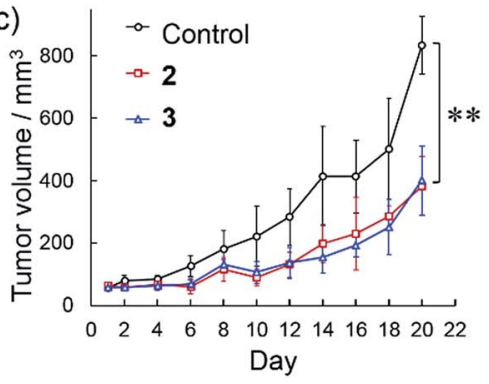

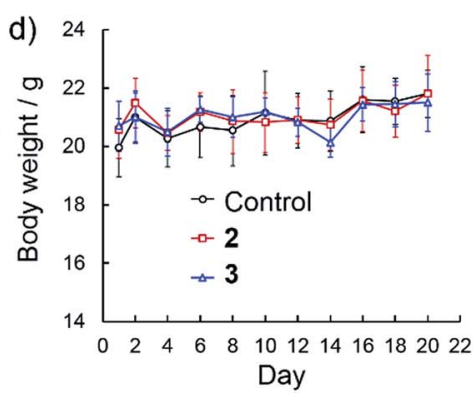

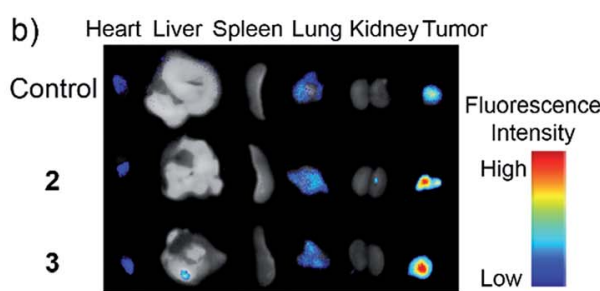

e)

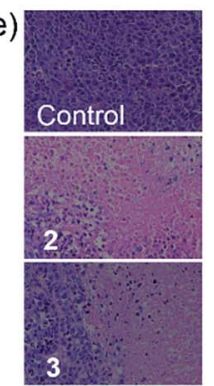

Fig. 5 In vivo tumor imaging and antitumor study via intravenous injection of NanoTPES: ( $a$ and b) body imaging and tissue imaging of the distribution of NanoTPES after intravenous injection. Black circles indicate tumor sites; (c) tumor volume up to day 20 after treatment with NanoTPES 2 and 3 (mean \pm s.d., $n=5$ ) (**P<0.01; Student's $t$-test); (d) variation of the body weight of mice up to day 20 after treatment; (e) H\&E analysis of tumor tissues from mice treated with PBS (1\% DMSO), NanoTPES 2 and 3 for 20 days, respectively. 
NanoTPES 2 or 3 (Fig. 3b). NanoTPES 2 and 3 also displayed high cytotoxicity toward cancerous HeLa and A549 cells. The cell viabilities were only $23 \%$ and $8 \%$ for HeLa cells, and $23 \%$ and $31 \%$ for A549 cells after the respective treatments with NanoTPES 2 and 3 (Fig. S14 $\dagger$ ). In comparison, normal human cells including HEK293, Chang liver and L02 cells were healthy and almost unaffected after the same treatments (Fig. 3a and $\mathrm{S} 14 \dagger$ ), and in each case the cell viability was greater than $90 \%$. Clearly, NanoTPES 2 and 3 exhibit high cytotoxicity towards cancer cells with almost no cytotoxicty towards normal cells. The $\mathrm{IC}_{50}$ values of NanoTPES 2 and 3 towards HepG2 cells were estimated to be $2.2 \mu \mathrm{M}$ and $3.8 \mu \mathrm{M}$, respectively (Fig. S15 $\dagger$ ). In contrast, NanoTPES $\mathbf{1}$ is cytotoxic towards both cancer and normal cells. Cell viabilities were found to be below $50 \%$ for both cancer (HepG2, HeLa and A549) and normal (HEK293, Chang liver and L02) cells (Fig. 3a and S14†). NanoTPES 4, however, shows no cytotoxicity towards both cancer and normal cells.

\section{Mechanism for the tunable cytotoxicity}

Such tunable cytotoxicity of NanoTPES with different counter anions is in good agreement with the above confocal imaging results. To further investigate the roles of cations and anions for cytotoxic effects, two control salts 5 and 6 (Scheme 1), which contain tetraphenyl borate as the anion and $\mathrm{Na}^{+}$and 1-methyl-4phenylpyridinium as the respective cations, were prepared. The results reveal that both 5 and $\mathbf{6}$ show negligible cellular uptake and no cytotoxicity to HepG2 cells based on the MTT assay (Fig. S16†). Therefore, it can be inferred that the cation, pyridinium-substituted tetraphenylethylene, and the respective anions within NanoTPES 2 and 3 work cooperatively to achieve targeted imaging and selective cytotoxicity. Pyridiniumsubstituted tetraphenylethylene plays a central role in their cytotoxicities, while different anions tune the cytotoxicities to be selective.

Further studies indicate that the accumulation of NanoTPES 2 and $\mathbf{3}$ in mitochondria of cancer cells can induce a change of membrane potential $\left(\Delta \Psi_{\mathrm{m}}\right)$, which is an indicator of the health status of mitochondria and is closely related with early cell apoptosis. $\Delta \Psi_{\mathrm{m}}$ was measured with a commercial JC-10 dye. From both CLSM observation and microplate quantitation assay, a critical drop in $\Delta \Psi_{\mathrm{m}}$ of HepG2 after treatment with NanoTPES 2 and 3 was detected (Fig. S17, ESI $\dagger$ ). These results hint that the selective cytotoxicity of NanoTPES 2 and 3 towards HepG2 cells may operate via the mitochondria damage-mediated cell apoptosis pathway as illustrated in Scheme 2. Additionally, NanoTPES $\mathbf{2}$ and $\mathbf{3}$ were also found to affect the generation of adenosine triphosphate (ATP) in HepG2 cells as indicated by an ATP bioluminescent assay kit. The amounts of ATP generated in HepG2 cells after incubation with NanoTPES 2 and 3 decreased by $67 \%$ and $57 \%$ of that before the treatments based on the relative emission intensities (Fig. S17†). These results suggest that NanoTPES 2 and 3 can inhibit the oxidative phosphorylation process in mitochondria. The reduced ATP supply in the mitochondria of HepG2 cells finally leads to cell death.

\section{In vivo xenografted tumor therapy}

Benefiting from their cancer cell-specific internalization and mitochondria-damaging ability, NanoTPES 2 and NanoTPES 3 were applied for in vivo antitumor therapy. To this end, a tumorbearing mouse model was established by xenografting HepG2 cells into BALB/c nude mice subcutaneously. After tumor initiation, PBS containing 1\% DMSO (v/v), NanoTPES 2 and NanoTPES 3 were administrated separately by either intratumor or intravenous injection every other day.

After intratumor injection of NanoTPES 2 and 3, the mice showed significantly lower tumor growth rates than control ones administrated with PBS (1\% DMSO) (Fig. 4a). With 20 day treatment, the average tumor volumes were $217 \mathrm{~mm}^{3}$ and $310 \mathrm{~mm}^{3}$, respectively, being much smaller than that of the control group $\left(587 \mathrm{~mm}^{3}\right)$. Clearly, the rate of tumor growth is slowed significantly after the respective treatments with NanoTPES 2 and 3. This agrees well with the actual photos of tumors after 20 day treatments (see Fig. S18 $\dagger$ ). The body weight of mice is used to evaluate the side effects. Compared with control mice, their weights remained almost unchanged (Fig. 4b), suggesting that NanoTPES 2 and 3 possess minimal systemic cytotoxicity. To further evaluate the therapeutic efficacy, hematoxylin and eosin (H\&E) histopathological analysis was employed. Distinct cell death was observed in the tumor tissues treated by NanoTPES 2 and 3, while tumor cells in the control group retained vigorous and cancerous features (Fig. S18 $\dagger$ ).

The targetable antitumor efficacy was further examined by intravenous injection of NanoTPES 2 and 3 to tumor-bearing mice. Body imaging was carried out by monitoring the respective red emissions. As shown in Fig. 5a, significant fluorescence was detected in the tumors after intravenous injections of NanoTPES 2 and 3 separately, indicating the specific delivery of these nanoparticles to the tumor sites. After sacrifice, bright red emission was clearly observed in tumor tissues (Fig. 5b), while other organs including the heart, liver, spleen, lung and kidney showed negligible emissions. Such in vivo tumor-targeting and accumulation abilities of NanoTPES 2 and 3 are appealing for their antitumor performance.

The in vivo therapeutic outcomes after intravenous injection of NanoTPES 2 and 3 are shown in Fig. 5c-e. In comparison with those of the control group, the tumor volumes were obviously reduced after injections of these two nanoparticles. The average volumes of the tumors after intravenous injections of NanoTPES 2 and 3 for 20 days were $383 \mathrm{~mm}^{3}$ and $401 \mathrm{~mm}^{3}$, respectively, which were reduced by $54 \%$ and $52 \%$, respectively, in comparison with that $\left(834 \mathrm{~mm}^{3}\right)$ of the control group. Moreover, the NanoTPES-treated mice did not show significant loss in body weight (Fig. 5d), suggesting intravenous injections of NanoTPES 2 and 3 have almost no obvious side toxicity. H\&E histopathological analysis indicates that tumor cell death was only observed for mice treated with NanoTPES 2 and 3 (Fig. 5e). This further verifies their selective toxicity to cancer cells. No obvious physiological morphology changes were found in the heart, liver, spleen, lung and kidney (Fig. S19†), demonstrating the negligible side effect of NanoTPES 2 and 3 during blood circulation. The tumor targeting effect and cancer cell-specific 
damage capability provides NanoTPES 2 and 3 with superior therapeutic outcome and minimal systemic toxicity. Notably, such antitumor function has never been reported for AIE luminogens before.

\section{Conclusions}

In summary, we report the nanoparticles NanoTPES 1-4, derived from pyridinium-substituted tetraphenylethylene with different counter anions, for bioimaging and selective cytotoxicity towards cancer cells. The results reveal that the emissions, sizes and surface charges of these nanoparticles can be tuned by varying the counter anions. The suitable sizes and positively charged surfaces enable NanoTPES 2 and $\mathbf{3}$ to selectively image and target mitochondria in cancer cells rather than normal cells. Furthermore, the targeting of mitochondria in cancerous HepG2 cells by either NanoTPES 2 or 3 induces the decrease of mitochondrial transmembrane potential and affects ATP generation, thus resulting in mitochondrial damage (Scheme 2). In this manner, both NanoTPES 2 and 3 show selective cytotoxicity towards cancer cells, whilst they are almost nontoxic to normal cells. Inspired by this discovery, both NanoTPES 2 and 3 were applied for in vivo xenografted tumor therapy. To our delight, they specifically accumulate in tumor sites and exhibit efficient tumor suppression during in vivo therapy, whereas their systemic toxicity is negligible. These interesting results clearly demonstrate that the combination of pyridiniumsubstituted tetraphenylethylene and appropriate anions can lead to new multifunctional nanomaterials which can be used simultaneously for bioimaging and as antitumor agents.

\section{Acknowledgements}

Financial support from the National Natural Science Foundation of China (21375134, 21475140, 21135006 and 21321003), the Ministry of Science and Technology of China (2015CB856300, 2013CB733700, 2013CB834700), the Beijing Natural Science Foundation (2162048) and the Chinese Academy of Sciences (XDB12010300) is gratefully acknowledged.

\section{Notes and references}

1 (a) G. Szakács, J. K. Paterson and J. A. Ludwig, Nat. Rev. Drug Discovery, 2006, 5, 219; (b) X. Wang and Z. Guo, Chem. Soc. Rev., 2013, 42, 202.

2 (a) L. Gu and D. J. Mooney, Nat. Rev. Cancer, 2016, 16, 56; (b) F. Danhiera, O. Feronb and V. Préat, J. Controlled Release, 2010, 148, 135; (c) N. Bertrand, J. Wu, X. Xu, N. Kamaly and O. C. Farokhzad, Adv. Drug Delivery Rev., 2014, 66, 2.

3 (a) R. A. Petros and J. M. DeSimone, Nat. Rev. Drug Discovery, 2010, 9, 615; (b) H. Maeda, J. Wu, T. Sawa, Y. Matsumura and K. Hori, J. Controlled Release, 2000, 65, 271; (c) E.-K. Lim, T. Kim, S. Paik, S. Haam, Y.-M. Huh and K. Lee, Chem. Rev., 2015, 115, 327.

4 T. M. Allena and P. R. Cullis, Adv. Drug Delivery Rev., 2013, 65, 36.
5 (a) S. Kolemen, T. Ozdemir, D. Lee, G. M. Kim, T. Karatas, J. Yoon and E. U. Akkaya, Angew. Chem., Int. Ed., 2016, 55, 1; (b) A. Riedinger, T. Avellini, A. Curcio, M. Asti, Y. Xie, R. Tu, S. Marras, A. Lorenzoni, S. Rubagotti, M. Iori, P. C. Capponi, A. Versari, L. Manna, E. Seregni and T. Pellegrino, J. Am. Chem. Soc., 2015, 137, 15145; (c) M. E. Davis, Z. G. Chen and D. M. Shin, Nat. Rev. Drug Discovery, 2008, 7, 771.

6 (a) M. Ferrari, Nat. Rev. Cancer, 2005, 5, 161; (b) T. Sun, Y. S. Zhang, B. Pang, D. C. Hyun, M. Yang and Y. Xia, Angew. Chem., Int. Ed., 2014, 53, 12320.

7 (a) D. K. Bwambok, B. El-Zahab, S. K. Challa, M. Li, L. Chandler, G. A. Baker and I. M. Warner, ACS Nano, 2009, 3, 3854; (b) P. K. S. Magut, S. Das, V. E. Fernand, J. Losso, K. McDonough, B. M. Naylor, S. Aggarwal and I. M. Warner, J. Am. Chem. Soc., 2013, 135, 15873; (c) B. Wang, H. Yuan, Z. Liu, C. Nie, L. Liu, F. Lv, Y. Wang and S. Wang, Adv. Mater., 2014, 26, 5986; (d) C. Zhu, L. Liu, Q. Yang, F. Lv and S. Wang, Chem. Rev., 2012, 112, 4687; (e) A. Shao, Y. Xie, S. Zhu, Z. Guo, S. Zhu, J. Guo, P. Shi, T. James, H. Tian and W. Zhu, Angew. Chem., Int. Ed., 2015, 54, 7275; (f) Y. Li, A. Shao, Y. Wang, J. Mei, D. Niu, J. Gu, P. Shi, W. Zhu, H. Tian and J. Shi, Adv. Mater., 2016, 28, 3187; $(g)$ W. Lin, T. Sun, Z. Xie, J. Gu and X. Jing, Chem. Sci., 2016, 7, 1846.

8 (a) J. Mei, N. L. C. Leung, R. T. K. Kwok, J. W. Y. Lam and B. Z. Tang, Chem. Rev., 2015, 115, 11718; (b) J. Mei, Y. Hong, J. W. Y. Lam, A. Qin, Y. Tang and B. Z. Tang, Adv. Mater., 2014, 26, 5429; (c) Y. Hong, J. W. Y. Lam and B. Z. Tang, Chem. Commun., 2009, 29, 4332; (d) Z. Zhao, J. W. Y. Lam and B. Z. Tang, J. Mater. Chem., 2012, 22, 23726. 9 (a) R. T. K. Kwok, C. W. T. Leung, J. W. Y. Lam and B. Z. Tang, Chem. Soc. Rev., 2015, 44, 4228; (b) M. Wang, G. Zhang, D. Zhang and D. Zhu, J. Mater. Chem., 2010, 20, 1858; (c) G. Zhang, F. Hu and D. Zhang, Langmuir, 2015, 31, 4593; (d) W. Chen, Q. Li, W. Zheng, F. Hu, G. Zhang, Z. Wang, D. Zhang and X. Jiang, Angew. Chem., Int. Ed., 2014, 53, 13734; (e) S. Gui, Y. Huang, F. Hu, Y. Jin, G. Zhang, L. Yan, D. Zhang and R. Zhao, Anal. Chem., 2015, 87, 1470.

10 (a) D. Ding, K. Li, B. Liu and B. Z. Tang, Acc. Chem. Res., 2013, 46, 2441; (b) Y. Huang, F. Hu, R. Zhao, G. Zhang, H. Yang and D. Zhang, Chem.-Eur. J., 2014, 20, 158; (c) F. Hu, Y. Huang, G. Zhang, R. Zhao, H. Yang and D. Zhang, Anal. Chem., 2014, 86, 7987; (d) X. Gu, E. Zhao, J. W. Y. Lam, Q. Peng, Y. Xie, Y. Zhang, K. S. Wong, H. H. Y. Sung, I. D. Williams and B. Z. Tang, Adv. Mater., 2015, 27, 7093; (e) L. Zhang, W. Liu, X. Huang, G. Zhang, X. Wang, Z. Wang, D. Zhang and X. Jiang, Analyst, 2015, 140, 5849; (f) N. Zhao, M. Li, Y. Yan, J. W. Y. Lam, Y. L. Zhang, Y. S. Zhao, K. S. Wong and B. Z. Tang, J. Mater. Chem., 2013, 1, 4640; (g) Y. Yuan, C.-J. Zhang, S. Xu and B. Liu, Chem. Sci., 2016, 7, 1862.

11 (a) K. Li, W. QinM, D. Ding, N. Tomczak, J. Geng, R. Liu, J. Liu, X. Zhang, H. Liu, B. Liu and B. Tang, Sci. Rep., 2013, 3, 1150; (b) K. Li, Y. Jiang, D. Ding, X. Zhang, Y. Liu, J. Hua, S. Feng and B. Liu, Chem. Commun., 2011, 47, 7323; (c) D. Ding, K. Li, W. Qin, R. Zhan, Y. Hu, J. Liu, B. Tang and B. Liu, Adv. Healthcare Mater., 2013, 2, 500; (d) 
G. Feng, W. Qin, Q. Hu, B. Z. Tang and B. Liu, Adv. Healthcare Mater., 2015, 4, 2667.

12 (a) S. E. Weinberg and N. S. Chandel, Nat. Chem. Biol., 2015, 11, 9; (b) G. Yu, D. Wu, Y. Li, Z. Zhang, L. Shao, J. Zhou, Q. Hu, G. Tang and F. Huang, Chem. Sci., 2016, 7, 3017.

13 (a) M. T. Villanueva, Nat. Rev. Cancer, 2015, 15, 456; (b) G. Kroemer and J. C. Reed, Nat. Med., 2000, 6, 513.

14 (a) C. Lee, H.-K. Park, H. Jeong, J. Lim, A.-J. Lee, K. Y. Cheon, C.-S. Kim, A. P. Thomas, B. Bae, N. D. Kim, S. H. Kim, P.-G. Suh, J.-H. Ryu and B. H. Kang, J. Am. Chem. Soc., 2015, 137, 4358; (b) K. Suntharalingam, S. G. Awuah, P. M. Bruno, T. C. Johnstone, F. Wang, W. Lin, Y.-R. Zheng, J. E. Page, M. T. Hemann and S. J. Lippard, J. Am. Chem. Soc., 2015, 137, 2967; (c) S. Imstepf,
V. Pierroz, R. Rubbiani, M. Felber, T. Fox, G. Gasser and R. Alberto, Angew. Chem., Int. Ed., 2016, 55, 2792.

15 (a) D. Y. Cho, H. Cho, K. Kwon, M. Yu, E. Lee, K. M. Huh, D. H. Lee and H. C. Kang, Adv. Funct. Mater., 2015, 25, 5479; (b) A. T. Hoye, J. E. Davoren, P. Wipf, M. P. Fink and V. E. Kagan, Acc. Chem. Res., 2008, 41, 87; (c) A. R. L. Gear, J. Biol. Chem., 1974, 249, 3628.

16 F. Hu, G. Zhang, C. Zhan, W. Zhang, Y. Yan, Y. Zhao, H. Fu and D. Zhang, Small, 2015, 11, 1335.

17 I. Brigger, C. Dubernet and P. Couvreur, Adv. Drug Delivery Rev., 2002, 54, 631.

18 (a) S. Ran, A. Downes and P. E. Thorpe, Cancer Res., 2002, 62, 6132; (b) P. Chandra, H.-B. Noh and Y.-B. Shim, Chem. Commun., 2013, 49, 1900. 